\title{
Delayed Onset of Manic Symptoms in a Patient with Influenza A (H1N1) after administration of Oseltamivir (Tamiflu): A Case Report
}

\author{
Min Jhon, Ju-Wan Kim, Hee-Ju Kang, Seon-Young Kim, Ju-Yeon Lee, Sung-Wan Kim, Il-Seon Shin, \\ Jae-Min Kim
}

Department of Psychiatry, Chonnam National University Medical School, Gwangju, Korea

\begin{abstract}
Psychiatric side effects of oseltamivir can result in accident-proneness and suicide. Reportedly, such adverse psychiatric events are more common in children than in adults, but other risk factors are not known. We present a 13-year-old girl with influenza infection who developed manic symptoms after taking oseltamivir and receiving the human papillomavirus vaccination. While other research has found that psychiatric side effects associated with oseltamivir generally occur within 48 hours after beginning administration, in this case the manic symptoms developed on the fourth day after cessation of 5-day course of oseltamivir administration. Based on our review of this case, we recommend that clinicians should carry out vigilant monitoring of each patient's mental state when the patient is young, has a family history of psychiatric disorder, has drug sensitivity and has received medical treatments such as vaccination before or after taking oseltamivir. In addition, as side effects of oseltamivir may occur more than 48 hours after administration, it will be necessary to observe patients for several days after the prescription of oseltamivir.
\end{abstract}

KEY WORDS: Oseltamivir; Influenza A virus; Papillomavirus vaccines; Drug-related side effects and adverse reactions.

\section{INTRODUCTION}

Oseltamivir is a viral neuraminidase enzyme inhibitor commonly prescribed as an effective treatment for influenza infection [1]. Common adverse effects of oseltamivir are gastrointestinal side effects such as nausea, vomiting, diarrhea, and abdominal pain, although these are well tolerated by most patients [2]. Recently, there have been reports of adverse neuropsychiatric events, which may lead to accident-proneness or suicide. Abnormal behaviors have been reported including delirious speech, frightening episodes, abrupt anger, abnormal overactivity leading to accidents, and putting unusual objects into the mouth [3]. There was a case of a depressive episode associated with oseltamivir use in a Korean female adolescent [4]. Another case study reported mood swings, nihilistic

Received: October 1, 2019/ Revised: October 30, 2019

Accepted: November 4, 2019

Address for correspondence: Jae-Min Kim

Department of Psychiatry, Chonnam National University Medical School, 160 Baekseo-ro, Dong-gu, Gwangju 61469, Korea

E-mail: jmkim@chonnam.ac.kr

ORCID: https://orcid.org/0000-0001-7409-6306 delusions and auditory hallucinations in a 22-year-old Korean male patient [5]. However, at the time of this report, there is little information in the literature regarding the presence of a relationship between Oseltamivir and manic episodes among those with influenza infection. Also, in most cases, psychiatric symptoms developed within two days of initiation of treatment. Here, we present a case of delayed onset mania associated with the use of oseltamivir in a 13-year old patient.

\section{CASE}

A 13-year-old girl developed fever and was diagnosed with influenza $A$ virus infection based on a positive result with a rapid detection kit in 2018. She took oral oseltamivir for 5 days and stopped taking the medication because her flu-like symptoms improved. Three days after discontinuing oseltamivir, she was given Gardasil, a human papillomavirus vaccine [6]. The next day, she developed significant psychiatric symptoms. According to her parents and her medical records, she had undergone normal development both physically and mentally. She had no

(c) This is an Open-Access article distributed under the terms of the Creative Commons Attribution Non-Commercial License (http://creativecommons.org/licenses/by-nc/4.0) which permits unrestricted non-commercial use, distribution, and reproduction in any medium, provided the original work is properly cited. 
problems with her family or friends and was well adjusted at school. Also, she had no personal history of psychiatric illness, but her aunt committed suicide, related to suspected bipolar symptoms.

The course of medication and her mood symptoms are presented in Figure 1. Beginning on the 9th day after the initial administration of oseltamivir, she presented with lability, talkativeness, increased self-esteem, flight of ideas, anxiety, and agitation. She had trouble falling asleep at the same time as the development of these other symptoms and the sleep problems worsened such that she could not sleep at all by the time she visited the hospital. The patient could not concentrate on her schoolwork and felt as if something was wrong with her. Additionally, she suffered from auditory hallucinations that were similar to whispering and beeping near her ears. She experienced severe distress due to these unwanted psychiatric symptoms, including the auditory hallucinations, and expressed a desire to commit suicide when the symptoms worsened. After telling her parents about her suicidal ideation, she visited our outpatient clinic on the 12th day after the initial administration of oseltamivir. She had no fever and other vital signs were normal. A blood test showed no abnormalities in her renal function, liver function, thyroid function or electrolytes. She had no seizures and her neurological examination showed intact functioning. There were no signs of intra- or extracranial causes of organic mental syndromes including delirium. To assess her manic symptoms, the Young Mania Rating Scale (YMRS) was administered during the first visit and her score was 20, suggesting severe mania [7]. For symptom management, we prescribed valproic acid $500 \mathrm{mg} /$ day, risperidone $0.5 \mathrm{mg} /$ day, and clonazepam $0.25 \mathrm{mg} /$ day. Her manic symptoms improved immediately after taking psychotropics but she presented depressive mood, distractibility, and suicidal ideation on the second visit. Also, she had rashes on her face and hands, which developed 2 days after starting the medicine. On the fifth day of the treatment, she and her parents said that symptoms were completely relieved except for mild depressive mood. We concluded that the remaining mild depressive mood was a normal reaction for a girl who has experienced sudden mood-related episode. Because her YMRS score was 3, we decided to discontinue the medication and observe the clinical manifestations. She was followed up 1 week after stopping medications, at which point, she had completely recovered and adjusted to her normal daily routine. The rashes were reduced but remained at the last visit. She had no further mood symptoms and remained psychologically stable 7 months after her last visit.

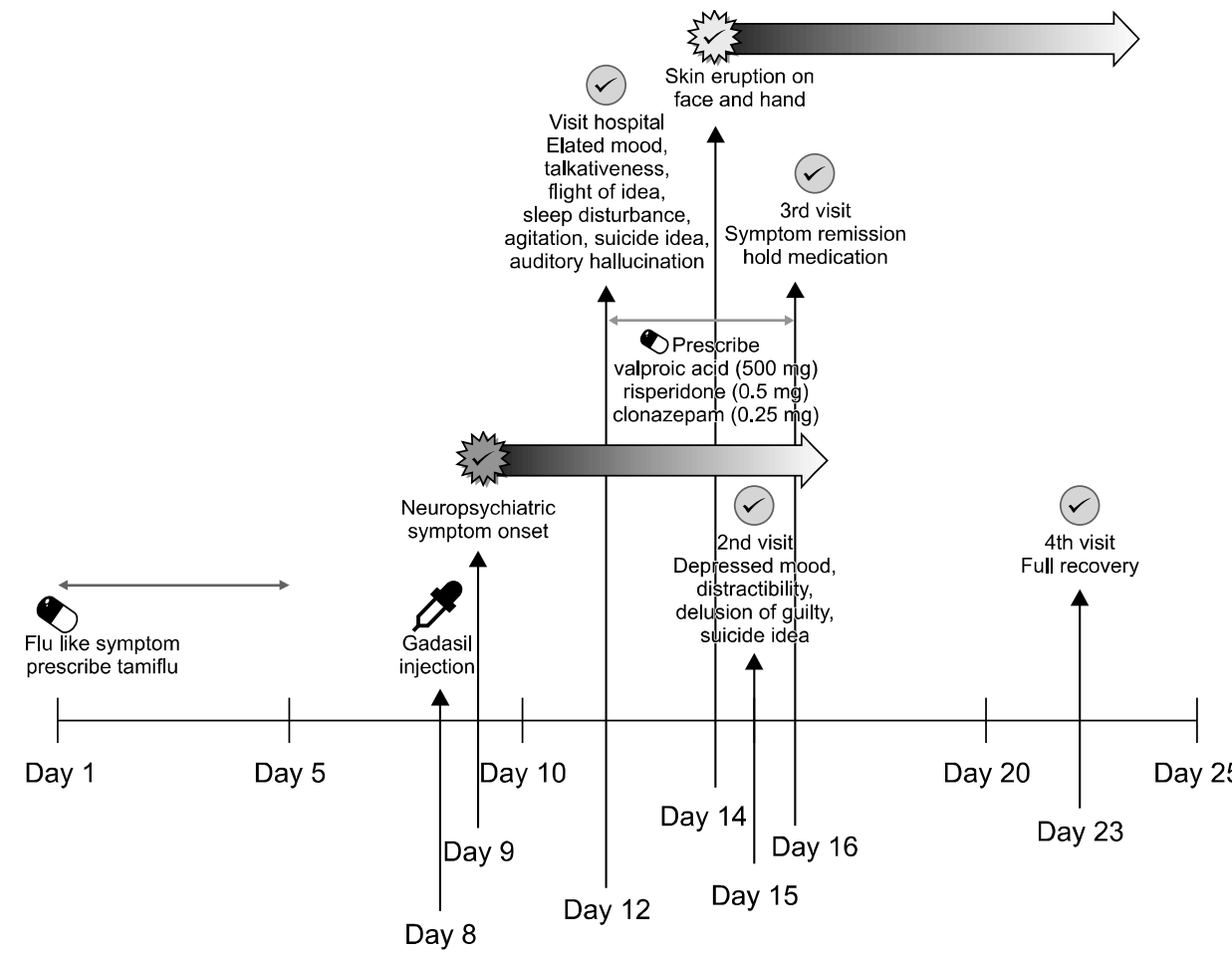

Fig. 1. Timeline of the illness, treatment and recovery of influenza A (H1N1) patient who took 5-day course of oseltamivir. 
After taking oseltamivir, our patient developed manic symptoms that caused clinically significant distress. However, her symptoms dramatically improved soon after the cessation of this medication. The patient had little evidence of independent bipolar and related disorder since she had no previous episodes of mood symptoms. There were no signs of delirium. Unfortunately, we did not perform brain imaging scans to evaluate the possibility of an organic issue in more detail. However, because the patient's vital signs, neurological examinations, and blood tests were normal, we assumed that her psychiatric symptoms were not related to an organic mental disorder. Thus, we concluded her final diagnosis was as substance/medication-induced bipolar and related disorder based on the criteria of the Diagnostic and Statistical Manual of Mental Disorders 5th edition.

\section{DISCUSSION}

We describe a patient with manic symptoms that followed oseltamivir intake to treat an influenza A ( $\mathrm{H} 1 \mathrm{~N} 1)$ infection. The pharmacological mechanism of the neuropsychiatric symptoms observed after oseltamivir administration is unknown. One study showed increased dopamine metabolism changes in the medial prefrontal cortices of rats after oseltamivir administration [8]. However, the effect of the influenza virus on the central nervous system is not fully understood, and it may also cause neuropsychiatric symptoms [9]. Toovey et al. [10,11] conducted an analysis in Japan, the United States, and other countries to estimate the incidence and characteristics of neuropsychiatric adverse events (NPAEs) in patients receiving oseltamivir. Their first review included 3,051 NPAEs between 1999 and September 15, 2007. The updated review included 1,805 NPAEs from September 16, 2007 to May 15, 2010. Both reviews concluded that the disease itself, rather than oseltamivir, is the more likely cause of such neuropsychiatric events.

Nevertheless, there are several reasons to believe that the manic symptoms of this patient may have resulted from taking oseltamivir. First, her manic symptoms developed soon after exposure to oseltamivir and persisted for a short period of time. Second, individuals her age are known to have more psychiatric side effects from medications than adults, and her symptoms have been reported in previous studies of psychiatric side effects asso- ciated with oseltamivir [11-13]. Third, she was highly responsive to mood stabilizers and antipsychotics, and completely recovered within 4 days. This suggests that her manic symptoms were caused by drugs rather than by non-medication-induced bipolar and related disorder. This step was important since she had a family history of bipolar disorder. Fourth, she presented manic symptoms after the flu-like symptoms and fever had subsided. Organic mental conditions such as encephalopathy related to influenza or delirium were ruled out by thorough interviews and assessments by a pediatric neurologist. Fifth, the occurrence of a skin rash shortly after starting psychiatric medications showed her sensitivity to the drugs. Development of psychiatric side effects of oseltamivir might have resulted from her drug sensitivity.

Our patient showed a number of differences in relation to previously described cases. First, it had been previously reported that psychiatric symptoms usually occur within 48 hours of oseltamivir intake [11]. As shown in Figure 1, our patient's psychiatric symptoms occurred on the fourth day after the end of 5 days of treatment with oseltamivir. Our hypothesis about this condition is that she might have a decreased ability to metabolize drugs. The persistence of her skin rash for more than a week after stopping the medication also suggests poor ability to metabolize the drugs. In addition, a case of neuropsychiatic symptoms the next day after a 5 day course of oseltamivir has been reported in Korea [5]. Second, most of the previous cases had no family history of psychiatric disorder, but our patient had an aunt who suffered from bipolar disorder. Given that manic symptoms as a side effect of oseltamivir are uncommon, it is plausible to suppose that the family history of bipolar disorder influenced the manifestation of psychiatric side effects. Third, she was injected with the Gardasil vaccine one day before psychiatric symptom onset. Although there is no case report of neuropsychiatric side effects associated with this vaccination, despite its extensive use in pediatric clinics, symptoms may be triggered by an antibody reaction. The relationship between the joint administration of oseltamivir and Gardasil and subsequent side effects should be studied.

We described a 13-year-old girl who developed delayed manic symptoms after the administration of oseltamivir and the Gardasil vaccination. The patient is considered to be at high risk for developing psychiatric side effects since she has family history of bipolar disorder and is 
suspected to be sensitive to the drugs. We recommend that clinicians be attentive to potential psychiatric side effects of oseltamivir, especially when the patient is young, has a family history of psychiatric disorders, has drug sensitivity and has received other medical treatment such as vaccination before or after taking oseltamivir. In addition, as the results of our study suggest that side effects of oseltamivir may occur several days after administration, it will be necessary to observe patients for some time after they stop taking it.

\section{- Acknowledgments}

This study was supported by a grant (BCRI 18018) Chonnam National University Hospital Biomedical Research Institute to J-MK.

\section{Conflicts of Interest}

No potential conflict of interest relevant to this article was reported.

\section{Author Contributions}

Conceptualization: Jae-Min Kim, Min Jhon, Ju-Wan Kim. Data acquisition: Min Jhon, Ju-Wan Kim. Formal analysis: Min Jhon, Ju-Wan Kim. Funding: Jae-Min Kim. Supervision: Jae-Min Kim. Writing-original draft: Min Jhon, Ju-Wan Kim. Writing-review \& editing: Jae-Min Kim, Min Jhon, Ju-Wan Kim, Hee-Ju Kang, Seon-Young Kim, Ju-Yeon Lee, Sung-Wan Kim, Il-Seon Shin.

\section{ORCID}

Min Jhon

Ju-Wan Kim

https://orcid.org/0000-0002-0408-768X

Hee-Ju Kang

Seon-Young Kim

Ju-Yeon Lee

Sung-Wan Kim

II-Seon Shin

Jae-Min Kim https://orcid.org/0000-0002-9888-1090

https://orcid.org/0000-0002-4784-4820

https://orcid.org/0000-0003-3185-9005

https://orcid.org/0000-0003-0653-7223

https://orcid.org/0000-0002-6739-2163

https://orcid.org/0000-0001-5370-7649

https://orcid.org/0000-0001-7409-6306

\section{REFERENCES}

1. Nicholson KG, Aoki FY, Osterhaus AD, Trottier S, Carewicz $\mathrm{O}$, Mercier $\mathrm{CH}$, et al. Efficacy and safety of oseltamivir in treatment of acute influenza: a randomised controlled trial. Neuraminidase Inhibitor Flu Treatment Investigator Group. Lancet 2000;355:1845-1850.

2. Dalvi PS, Singh A, Trivedi HR, Mistry SD, Vyas BR. Adverse drug reaction profile of oseltamivir in children. J Pharmacol Pharmacother 2011;2:100-103.

3. Yorifuji T, Suzuki E, Tsuda T. Oseltamivir and abnormal behaviors: true or not? Epidemiology 2009;20:619-621.

4. Chung S, Joung YS. Oseltamivir (tamiflu) induced depressive episode in a female adolescent. Psychiatry Investig 2010;7: 302-304.

5. Jeon SW, Han C. Psychiatric symptoms in a patient with influenza A (H1N1) treated with oseltamivir (tamiflu): a case report. Clin Psychopharmacol Neurosci 2015;13:209-211.

6. McLemore MR. Gardasil: introducing the new human papillomavirus vaccine. Clin J Oncol Nurs 2006; 10:559-560.

7. Lukasiewicz M, Gerard S, Besnard A, Falissard B, Perrin E, Sapin $\mathrm{H}$, et al. Young mania rating scale: how to interpret the numbers? Determination of a severity threshold and of the minimal clinically significant difference in the EMBLEM cohort. Int J Methods Psychiatr Res 2013;22:46-58.

8. Yoshino T, Nisijima K, Shioda K, Yui K, Kato S. Oseltamivir (tamiflu) increases dopamine levels in the rat medial prefrontal cortex. Neurosci Lett 2008;438:67-69.

9. Nakamura K, Schwartz BS, Lindegårdh N, Keh C, Guglielmo BJ. Possible neuropsychiatric reaction to high-dose oseltamivir during acute 2009 H1N1 influenza A infection. Clin Infect Dis 2010;50:e47-e49.

10. Toovey S, Prinssen EP, Rayner CR, Thakrar BT, Dutkowski R, Koerner A, et al. Post-marketing assessment of neuropsychiatric adverse events in influenza patients treated with oseltamivir: an updated review. Adv Ther 2012;29:826-848.

11. Toovey S, Rayner C, Prinssen E, Chu T, Donner B, Thakrar B, et al. Assessment of neuropsychiatric adverse events in influenza patients treated with oseltamivir: a comprehensive review. Drug Saf 2008;31:1097-1114.

12. Fuyuno I. Tamiflu side effects come under scrutiny. Nature 2007; 446:358-359.

13. Maxwell SR. Tamiflu and neuropsychiatric disturbance in adolescents. BMJ 2007;334:1232-1233. 